\title{
Acute Neurogenic Pulmonary Edema After Depth Electrode Placement for Epilepsy Surgery
}

\author{
F.D. Jacob, M.B. Wheatley, D.B. Sinclair
}

Can. J. Neurol. Sci. 2010; 37: 885-887

Acute neurogenic pulmonary edema (NPE) is a common and often under recognized disorder that can occur after an acute central nervous system injury ${ }^{1}$. Common causes include seizures, subarachnoid and intracerebral hemorrhage ${ }^{2}$, blunt or penetrating head trauma, and neurosurgical procedures ${ }^{3,4}$. The pathophysiology of NPE is still largely unknown, but it is believed to be the result of a localized brain injury, which induces an adrenergic response and subsequently acute pulmonary edema ${ }^{1}$. Neurogenic pulmonary edema presents clinically with the sudden onset of dyspnea, tachypnea, and tachycardia in the context of a central neurologic insult. Common mimic includes congestive heart failure, acute respiratory distress symptom and aspiration pneumonia. Chest radiographs typically show a normal size heart with evidence of pulmonary edema ${ }^{5,6}$. Neurogenic pulmonary edema is generally managed in a supportive and conservative fashion. The majority resolve within 48 to 72 hours ${ }^{7}$. The outcome of patients with NPE is usually determined by the course of the neurological insult and appropriate treatment or supportive measures. We present the case of a 13-year-old adolescent who developed acute neurogenic pulmonary edema after bilateral temporal lobe depth electrode placement for epilepsy surgery.

\section{CASE Report}

A 13-year-old male with a past medical history of intractable epilepsy was admitted electively to the Comprehensive Epilepsy Program at the University of Alberta for epilepsy surgery. He has a long history of complex partial seizures consisting of recurrent stereotypic episodes of altered level of consciousness, with preservation of speech and automatism. The seizures were intractable to medical management. His current medications included Valproic Acid $250 \mathrm{mg}$ once each morning and $500 \mathrm{mg}$ once each evening, and Clobazam $20 \mathrm{mg}$ twice a day. He was otherwise healthy, with no other medical conditions. Previous electro-encephalography (EEG) studies and Long Term Video EEG monitoring revealed independent interictal generalized, as well as right and left temporal epileptic discharges. On one occasion a right temporal lobe seizure onset was identified. Magnetic resonance imaging revealed a possible small illdefined lesion of the right amygdala, which could be consistent with a focal cortical dysplasia or a low-grade glioma. The patient was admitted electively for intracranial depth electrode seizure investigation. He was implanted with bitemporal depth electrodes, for confirmation of the location of the epileptic foci prior to undergoing a temporal lobe surgery. Bitemporal depth electrode placed orthogonally through the middle temporal gyrus using the Stealth Treon (Medtronic, USA neuronavigation system. Figure 1 shows the right temporal lobe depth electrode

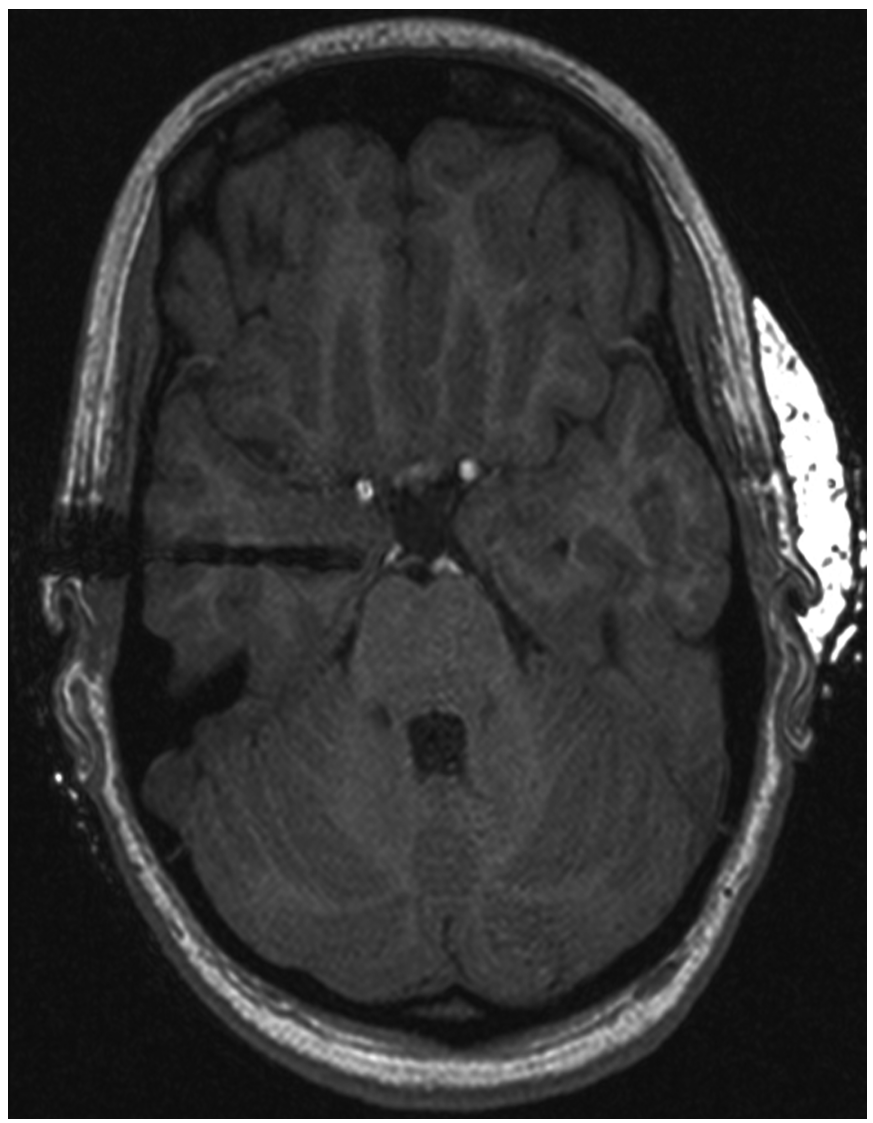

Figure 1: Axial T1-Weighted MRI of the head revealing one right temporal lobe depth electrode positioning.

position. During the procedure the patient suddenly became bradycardic, had bilateral pupillary dilation, and became tachypneic with frothy secretions from the endotracheal tube. This episode was felt to be a seizure and he was treated promptly with Dilantin and respiratory support. Because of the concern

From the Comprehensive Epilepsy Program, University of Alberta, Edmonton, Alberta, Canada.

Received March 16, 2010. Final Revisions Submitted June 24, 2010.

Correspondence to: D.B. Sinclair, Division of Pediatric Neurology, Department of Pediatrics, Stollery Children's Hospital, \#7319A Aberhart Centre 1, 11402 University Ave NW, Edmonton, Alberta, T6G 2J3, Canada. 


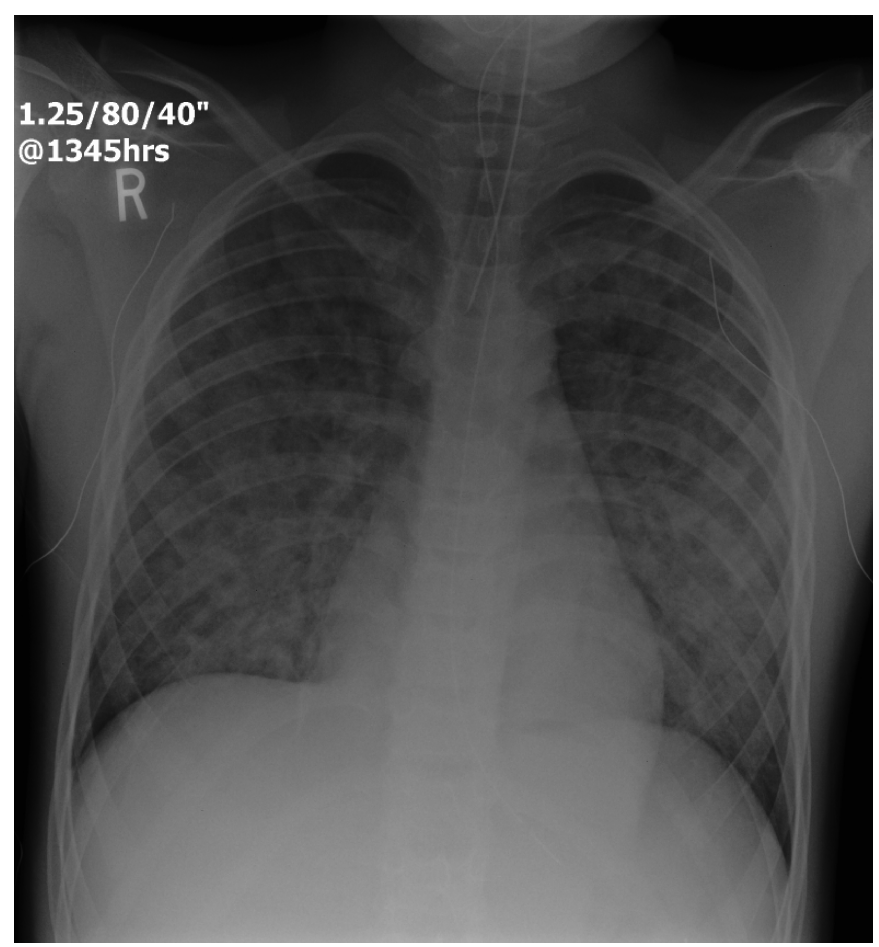

Figure 2: Chest-X-ray revealing bilateral consolidation sparing the costophrenic angles and apices, with a normal heart size and mediastenal contour. These findings are consistent with pulmonary edema.

over an intraoperative intracerebral hemorrhage secondary to the electrode placement, a computed tomogram (CT) scan of the head was performed acutely. It was normal and did not reveal any evidence of a hemorrhage. Due to increasing oxygen dependence he remained intubated and was transferred to the Pediatric Intensive Care Unit for ongoing management. A chest $\mathrm{X}$-ray revealed bilateral consolidation sparing the costophrenic angles and apices, with a normal heart size and mediastenal contour (Figure 2). The clinical history as well as the chest- X-ray was consistent with acute pulmonary edema. In the Pediatric Intensive Care Unit he required brief treatment with Epinephrine and Dobutamine and was started on Ceftriaxone for possible aspiration. Clinical status and cardiac function was monitored closely. His condition rapidly improved and he was extubated the next day. Antibiotic therapy was discontinued and he was gradually weaned off the oxygen over three days. On Day 3 post procedure he was back to his normal self and was transferred to the Pediatric Epilepsy Monitoring Unit for Long Term Video EEG monitoring. Numerous complex partial seizures were recorded, all of which originated in the right mesial temporal lobe (Figure 3). The depth electrodes were removed without complication and the patient was discharged from hospital. He subsequently underwent a right anterior temporal lobe resection. The pathology revealed a small foci of gliosis.

\section{Discussion}

Neurogenic pulmonary edema is a serious and life threatening condition occurring shortly after a neurological insult. The non- specific nature of the clinical signs of NPE make a definitive diagnosis difficult, and largely relies on the occurrence of pulmonary edema in the appropriate setting and in the absence of another obvious cause. Other causes that must be excluded include congestive heart failure and acute respiratory distress syndrome. The radiographic findings can be easily confused with aspiration pneumonia. In the presented case the event occurred suddenly in the operating room while the patient was intubated with no evidence of aspiration. The patient recovered rapidly and there was no evidence of cardiac dysfunction. Aspiration pneumonia tends to develop over a few days after the insult and last for one to two weeks, while NPE develops rapidly post insult and resolves within a few days. We believe that the depth electrode positioning in the right mesial temporal lobe led to NPE likely by provoking a seizure. A clinical event of paroxysmal bradycardia, tachypneia and bilateral pupillary dilatation occurred shortly after depth electrode implantation. This could be due to the insertion of the depth electrode or more likely due to a seizure precipitated by the electrode. Invasive intracranial monitoring using depth electrode as part of surgical epilepsy investigation is a common procedure at our institution, and this represents the first time we observed this complication. An association between depth electrode implantation and neurogenic pulmonary edema has never been reported in the literature. Complications of implantation have been reported in the order of $0.5-5 \%$ and most commonly involve an infection or the formation of a hematoma ${ }^{8-10}$. In the presented case, repeat CT scans were performed over 24 hours and did not show any evidence of hemorrhage. The association between seizures and neurogenic pulmonary edema is well recognized. Up to one-third of patients suffering from status epilepticus may present with symptoms of $\mathrm{NPE}^{11}$. In children it is believed that status epilepticus is the major cause of $\mathrm{NPE}^{12}$. Neurogenic pulmonary edema has also been linked to cases of sudden unexplained death in epilepsy. In a series of 52 patients with epilepsy and unexplained death, 45 had evidence of pulmonary edema on autopsy ${ }^{13}$. Interestingly in a recent study of epileptic baboons who died suddenly without an apparent cause, many had evidence of acute pulmonary edema ${ }^{14}$.

Seizures, especially mesial temporal lobe seizures, have long been associated with ictal autonomic changes, particularly cardiovascular changes, including tachycardia, bradycardia and arrhythmias. Frysinger et al, ${ }^{15}$ showed that neuronal discharges from the human mesial temporal structures, amygdala, and hippocampus are synchronized with the cardiac cycle and the respiratory cycle. Seizures arising from the temporal lobe also propagate more easily to the regulatory centers of the autonomic nervous system than extra temporal seizures. Leutmezer showed that in temporal lobe seizures ictal tachycardia preceded EEG seizure onset by 14 seconds and by 8 seconds in seizures of extratemporal origins ${ }^{16}$. The pathophysiology of NPE as stated earlier is still largely unknown, but is believed to be mediated via an acute activation of the sympathetic system and an adrenergic response to a CNS insult mediated via the medulla oblongata resulting in an increase in pulmonary hydrostatic pressure.

In summary our patient had an episode of acute neurogenic pulmonary edema during implantation of a depth electrode for epilepsy surgery. This case demonstrates that NPE is a possible complication of depth electrode implantation and highlights the importance for clinicians to be aware of this unusual syndrome. 


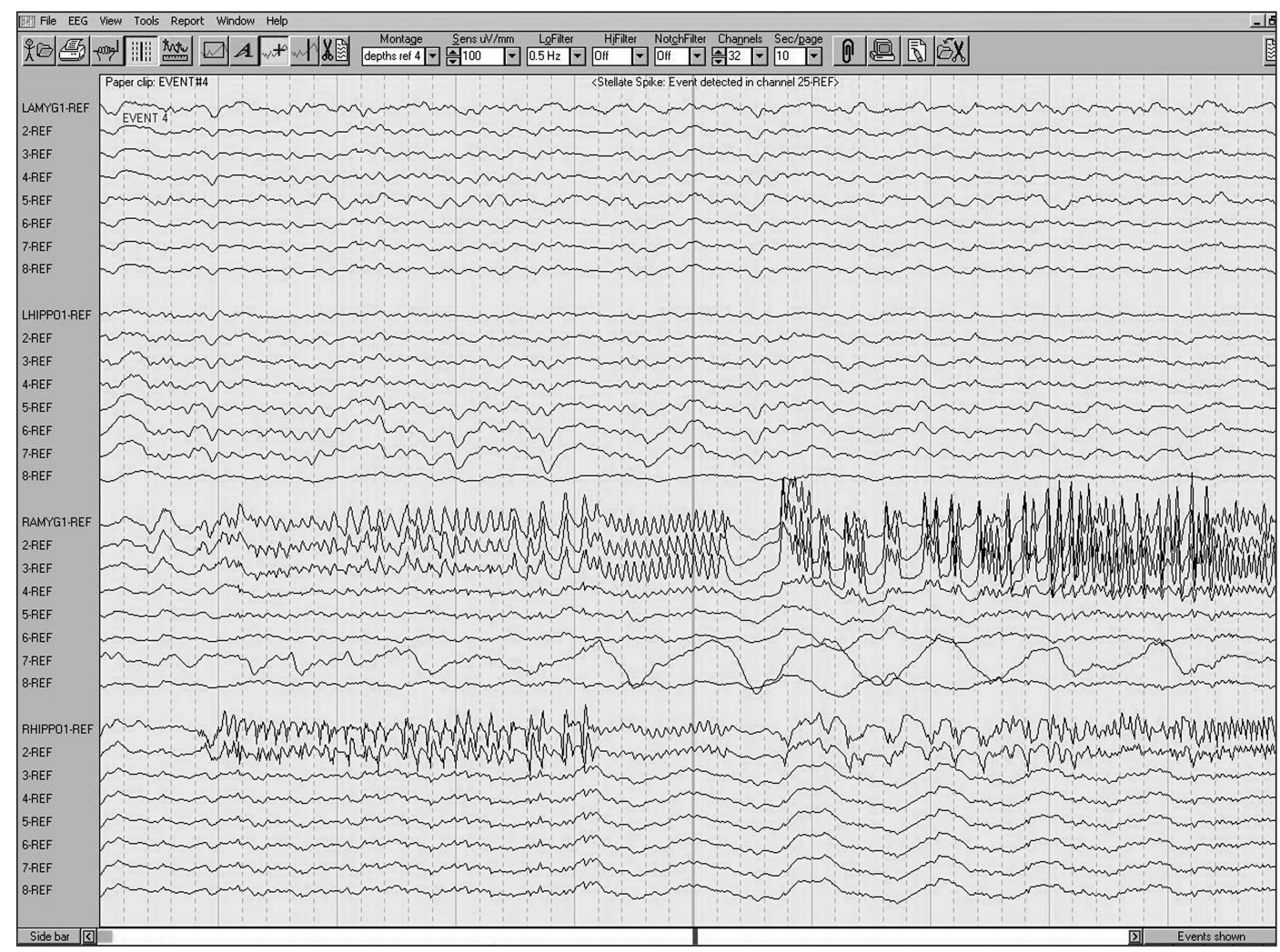

Figure 3: EEG of implanted bitemporal depth electrode. Focal seizures are recorded from the right temporal lobe, as identified in electrodes Right Amygdala and Right Hippocampus.

\section{REFERENCES}

1. Baumann A, Audibert G, McDonnell J, Mertes PM. Neurogenic pulmonary edema. Acta Anaesthesiol Scand. 2007 Apr;51(4): 447-55.

2. Mackersie RC, Christensen JM, Pitts LH, Lewis FR. Pulmonary extravascular fluid accumulation following intracranial injury. J Trauma. 1983 Nov;23(11):968-75.

3. Simmons RL, Martin AM Jr., Heisterkamp CA 3rd, Ducker TB. Respiratory insufficiency in combat casualties. II. Pulmonary edema following head injury. Ann Surg. 1969 Jul;170(1):39-44.

4. Colice GL, Matthay MA, Bass E, Matthay RA. Neurogenic pulmonary edema. Am Rev Respir Dis. 1984 Nov;130(5):941-8.

5. Ell SR. Neurogenic pulmonary edema. A review of the literature and a perspective. Invest Radiol. 1991 May;26(5):499-506.

6. Fontes RB, Aguiar PH, Zanetti MV, Andrade F, Mandel M, Teixeira MJ. Acute neurogenic pulmonary edema: case reports and literature review. J Neurosurg Anesthesiol. 2003 Apr;15(2): 144-50.

7. Lagerkranser M, Pehrsson K, Sylven C. Neurogenic pulmonary oedema. A review of the pathophysiology with clinical and therapeutic implications. Acta Med Scand. 1982;212(4):267-71.

8. Ojemann GA EJJ. Acute and chronic intracranial recording and stimulation. In: Engel J, editor. Surgical treatment of the epilepsies. New York: Raven Press; 1987. p. 263-88.
9. So N. Depth electrode studies in mesial temporal epilepsy. In: Luders H, editor. Epilepsy surgery. New York: Raven Press; 1992. p. 371-84.

10. Ross DA, Brunberg JA, Drury I, Henry TR. Intracerebral depth electrode monitoring in partial epilepsy: the morbidity and efficacy of placement using magnetic resonance image-guided stereotactic surgery. Neurosurgery. 1996 Aug;39(2):327-33; discussion 333-4.

11. Simon RP. Neurogenic pulmonary edema. Neurol Clin. 1993 May; 11(2):309-23.

12. Mulroy JJ, Mickell JJ, Tong TK, Pellock JM. Postictal pulmonary edema in children. Neurology. 1985 Mar;35(3):403-5.

13. Leestma JE, Walczak T, Hughes JR, Kalelkar MB, Teas SS. A prospective study on sudden unexpected death in epilepsy. Ann Neurol. 1989 Aug;26(2):195-203.

14. Szabo CA, Knape KD, Leland MM, et al. Mortality in captive baboons with seizures: a new model for SUDEP? Epilepsia. 2009 Aug;50(8):1995-8.

15. Frysinger RC, Harper RM. Cardiac and respiratory correlations with unit discharge in human amygdala and hippocampus. Electroencephalogr Clin Neurophysiol. 1989 Jun;72(6):463-70.

16. Leutmezer F, Schernthaner C, Lurger S, Potzelberger K, Baumgartner C. Electrocardiographic changes at the onset of epileptic seizures. Epilepsia. 2003 Mar;44(3):348-54. 